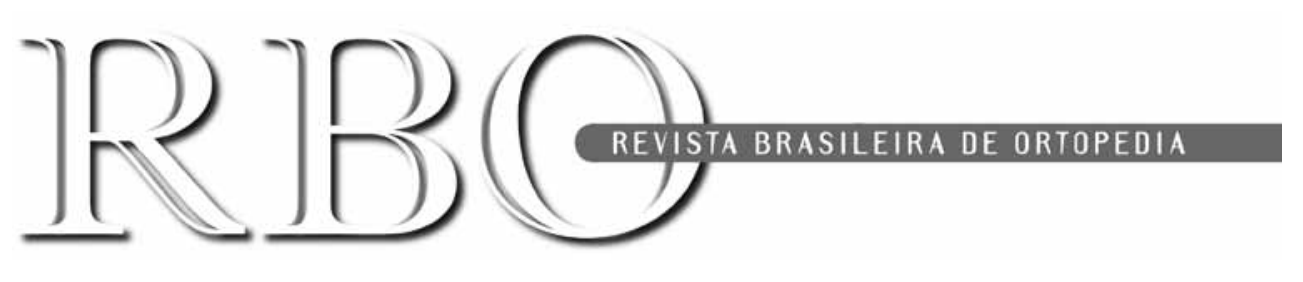

\title{
Editorial
}

\section{WILL THERE BE A FUTURE FOR THE RBO?}

\author{
Gilberto Luis Camanho
}

Thinking about the future of the RBO automatically leads to reflection on the future of our specialty, since it is certain that the RBO will be responsible for publicizing this future.

Taking a light but critical tone, I reflected on the progress of orthopedics over the last few years, by asking:

In what way have we evolved? Is there much to be done?

Recently, we commemorated the hundredth anniversary of the first description of aseptic necrosis of the femoral head in children, a disease that bears the name of its discoverers (at least four of them) and is extremely well known among our residents, since it is an obligatory term in exams.

Except for the variety of classifications and, consequently, questions for exams, there has not been any significant advance in treating the disease.

Fractures of the femoral neck, which have been and continue to be a central topic at various congresses, are still a subject for discussion regarding the best therapeutic method, especially for patients in their sixth decade. Doubts remain. The same is seen in relation to 
fractures of the proximal third of the tibia, which are also common within this age group. For such fractures, the traditional angled plate is still the best therapeutic option.

Fractures in general, and especially diaphyseal fractures, which now bear names that are numbers, have gone from absolute stability with enormous plates and many screws to relative stability with plates placed subcutaneously. However, except for the new and impenetrable nomenclature and the incredible variety of orifices and screws for plate fixation, the incidence of pseudarthrosis has changed very little.

Reconstruction of the anterior cruciate ligament is the subject with the largest number of published papers in the entire field of orthopedics. With more than 10,000 references in PubMed, it went through the negation of its importance at the time of the extraarticular reconstructions to come to today, when anatomical intra-articular reconstruction is proclaimed as a great novelty. Recently, a colleague who is not a knee specialist asked me: "But have you ever done any reconstructions that were not anatomical?".

Lesions of the joint cartilage, a topic that perhaps currently accounts for the greatest investment in hours of study, continues to have the same perspective that Sir William Hunter left us with, 300 years ago (1743), i.e. the perspective of an incurable condition. The drugs that brought so much hope seem to have little or no effect; and the results from surgical procedures, no matter how modern they may be, differ very little from the classical perforations or microfractures described by Pride more than 50 years ago.

Arthroplasty was a major advance in treating severe joint lesions. When this technique started to be used, 40 years ago, it presented serious problems that provoked fear among patients and surgeons alike: loosening and infection. Except for the duration of use and cost of antibiotics, there has been little change in the incidence of loosening and infection and in their treatment.

It would be unfair not to place value on advances in some areas, such as spinal surgery, arthroscopy and treatments for muscle-tendon injuries. Through the cost of performing spinal surgery, it has brought advances both for orthopedics and for metallurgy, since such surgery has led to the discovery of a metal that is more expensive than gold: the metal used for making pedicle screws.

Arthroscopy, which is greatly indicated, has made it possible to perform useless minimally invasive surgery: a fantastic advance providing protection for clients. Studies on treatments for muscle-tendon injuries have brought the great advance of a new use for blood. Until the time of these studies, it served only to treat anemia and hemorrhage, but it has now also come into use for local treatment of tendinitis and muscle injuries through platelet growth factors.

I was very happy at the end of these reflections, since I am sure that there will be material for the RBO for many years to come. 